\title{
Parity and valley degeneracy in multilayer graphene
}

\author{
Mikito Koshino ${ }^{1}$ and Edward McCann ${ }^{2}$ \\ ${ }^{1}$ Department of Physics, Tokyo Institute of Technology, 2-12-1 Ookayama, Meguro-ku, Tokyo 152-8551, Japan \\ ${ }^{2}$ Department of Physics, Lancaster University, Lancaster LA1 4YB, United Kingdom
}

(Received 17 December 2009; revised manuscript received 15 February 2010; published 12 March 2010)

\begin{abstract}
We study spatial symmetry in general $A B A$-stacked multilayer graphene to illustrate how electronic spectra at the two valleys are related in a magnetic field. We show that the lattice of multilayers with an even number of layers as well as that of monolayer graphene satisfy spatial inversion symmetry, which rigorously guarantees valley degeneracy in the absence of time-reversal symmetry. A multilayer with an odd number of layers (three or more) lacks inversion symmetry, but there is another transformation imposing an approximate valley degeneracy, which arises because the low-energy Hamiltonian consists of separate monolayerlike and bilayerlike parts. We show that an external electrostatic potential generally breaks valley degeneracy in a magnetic field, in a markedly different manner in odd and even multilayers.
\end{abstract}

DOI: 10.1103/PhysRevB.81.115315

PACS number(s): 73.22.Pr, 81.05.ue, 73.43.Cd

\section{INTRODUCTION}

The fabrication of individual graphene flakes, ${ }^{1}$ followed by an observation of the integer quantum Hall effect in them, ${ }^{2-4}$ triggered an explosion of interest in the electronic properties of graphene. It was fuelled, in part, by the realization that the low-energy band structure of a graphene monolayer consists of two Dirac cones centered at inequivalent corners of the Brillouin zone, $K_{+}$and $K_{-}$, which are called valleys [Fig. 1(b)]. They support chiral quasiparticles with opposite chirality in each valley and a linear dispersion reminiscent of the quantum electrodynamics of massless fermions. ${ }^{5-7}$ In the presence of time-reversal symmetry, the energy spectrum is degenerate between the different valleys since the time-reversal operation connects electronic states at $K_{+}$to those at $K_{-}$. In graphene, not only time-reversal symmetry, but parity, i.e., spatial inversion symmetry with respect to the center of a hexagon, ${ }^{7-9}$ is also able to transform electronic states between valleys. In the presence of a magnetic field, parity ensures degeneracy of the electronic spectra at different valleys.

In this paper, we study spatial symmetry in general $A B A$-stacked (Bernal) multilayer graphene composed of $N$ layers to illustrate how the electronic spectra at the two valleys are related in a magnetic field. In multilayers with even $N$, including bilayers, ${ }^{4,10,11}$ the lattice obeys spatial inversion symmetry $(x, y, z) \rightarrow(-x,-y,-z)$ similarly to monolayers, which swaps electronic states between valleys ensuring valley degeneracy in the absence of time-reversal symmetry. The picture is different in multilayers with odd $N$, starting from trilayer graphene $(N=3)$, because their lattices do not satisfy spatial inversion symmetry ${ }^{9,12}$ [Fig. 1(c)]. Here we consider an additional transformation which imposes an approximate valley degeneracy on the electronic spectra of odd- $N$ multilayers. It arises because the electronic Hamiltonian may be decomposed into separate monolayerlike and bilayerlike parts, ${ }^{13-16}$ with each part satisfying an approximate inversion symmetry corresponding to that of the real lattice of monolayer or bilayer graphene, respectively. We predict a peculiar Landau-level spectra in bilayer and trilayer graphenes in the presence of interlayer potential asymmetry, with unusual structures of broken valley degeneracy that are markedly different from each other.

\section{SYMMETRY ANALYSIS OF THE ELECTRONIC STRUCTURE}

We present here general symmetry arguments without referring to model-specific details in order to provide information about the valley degeneracy of Landau levels. We consider Bernal-stacked multilayer graphene with $N$ layers in an external uniform magnetic field $\mathbf{B}$ with arbitrary direction and an external potential $U(\mathbf{r})$ (other than the lattice poten- (a)

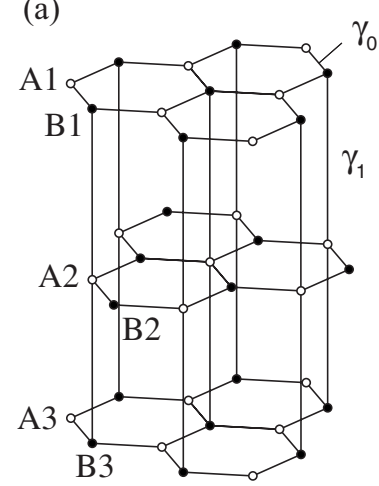

(c)

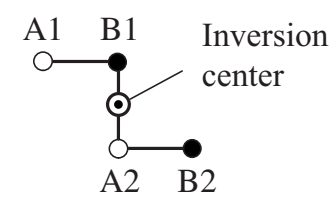

Bilayer (b)

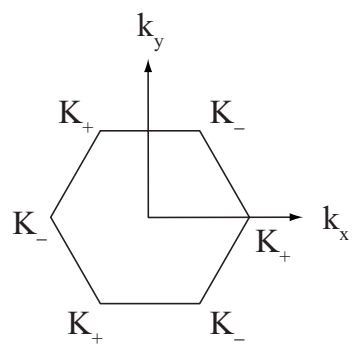

Brillouin zone
FIG. 1. (a) Atomic structure of $A B A$-multilayer graphene. (b) Schematic of the Brillouin zone with two inequivalent valleys $K_{+}$. (c) Schematic of the lattice of bilayer graphene (left) and of Bernalstacked trilayer graphene (right), with the inversion center for bilayer and the mirror plane for trilayer, respectively. 
tial) where $\mathbf{r}=(x, y, z)$. Without resorting to any approximations, we can formally express the total Hamiltonian of the system as $H[\mathbf{B}, U(\mathbf{r})]$ and we take $x$ and $y$ axes to be parallel to the graphene layers and the $z$ axis in the perpendicular direction.

Even- $N$ multilayers. In multilayers with an even number of layers, the lattice is symmetric with respect to spatial inversion symmetry $P[(x, y, z) \rightarrow(-x,-y,-z)]$ as shown in Fig. 1(c) because the point group of the lattice $D_{3 d}$ (Refs. 9 and 12) $\left(\left\{E, 2 C_{3}, 3 C_{2}^{\prime}, i, 2 S_{6}, 3 \sigma_{d}\right\}\right)$ can be regarded as a direct product of group $D_{3}\left(\left\{E, 2 C_{3}, 3 C_{2}^{\prime}\right\}\right)$ with the inversion group $C_{i}(\{E, i\})$. In the absence of $\mathbf{B}$, there is a symmetry with respect to time reversal $T(t \rightarrow-t)$. The influence of $P$ and $T$ on the Hamiltonians of even- $N$ multilayers is summarized as

$$
\begin{gathered}
P: P H[\mathbf{B}, U(\mathbf{r})] P^{-1}=H[\mathbf{B}, U(-\mathbf{r})], \\
T: H^{*}[\mathbf{B}, U(\mathbf{r})]=H[-\mathbf{B}, U(\mathbf{r})] .
\end{gathered}
$$

The magnetic field $\mathbf{B}$ does not change sign under the operation $P$ because it is an axial vector. Manes et al. ${ }^{9}$ used the combined $P T$ operation to show that the Fermi points are stable with respect to the opening of a gap.

In multilayer graphenes, electronic properties are well described by a $\mathbf{k} \cdot \mathbf{p}$ approximation in the vicinity of $K_{+}$and $K_{-}$ points. ${ }^{17}$ We can consider the $\mathbf{k} \cdot \mathbf{p}$ Hamiltonian (and thus eigenstates) at each valley separately, as long as the potential $U(\mathbf{r})$ is smooth in the $x y$ plane compared to the atomic scale and the magnetic field is not too strong as to mix states around the two valleys. When $\mathbf{B}=0$, the time-reversal symmetry in Eq. (2) ensures valley degeneracy of the electronic spectrum because the operation $T$ swaps eigenstates at $K_{+}$ and those of $K_{-}$through flipping the Bloch factor as $\left(e^{i \mathbf{K}_{ \pm} \cdot \mathbf{r}}\right)^{*}=e^{i \mathbf{K}_{\mp} \cdot \mathbf{r}}$. Here, $\mathbf{K}_{\xi}(\xi= \pm)$ are the wave numbers corresponding to $K_{\xi}$ points. ${ }^{17}$ The operation of spatial inversion $P$ also exchanges $K_{+}$and $K_{-}$because the point $-\mathbf{K}_{+}$is equivalent to $\mathbf{K}_{-}$in the Brillouin zone. Then, the symmetry of Eq. (1) suggests that the eigenstates of $K_{+}$at $[\mathbf{B}, U(\mathbf{r})]$ are related to those of $K_{-}$at $[\mathbf{B}, U(-\mathbf{r})]$. From this, we immediately conclude that the Landau levels in even- $N$ multilayers are degenerate in valleys as long as $U(\mathbf{r})=U(-\mathbf{r})$ or the external potential has inversion symmetry with the same symmetry point as that of the lattice potential.

Odd- $N$ multilayers. In odd- $N$ multilayers with $N \geq 3$, the point group $D_{3 h}\left(\left\{E, 2 C_{3}, 3 C_{2}^{\prime}, \sigma_{h}, 2 S_{3}, 3 \sigma_{v}\right\}\right)$ (Refs. 9 and 12) can be regarded as a direct product of the group $D_{3}\left(\left\{E, 2 C_{3}, 3 C_{2}^{\prime}\right\}\right)$ with the reflection group $C_{s}\left(\left\{E, \sigma_{h}\right\}\right)$. With respect to the even- $N$ multilayers, spatial inversion is replaced by mirror reflection $\sigma_{h}[(x, y, z) \rightarrow(x, y,-z)]$ as shown in Fig. 1(c), which does not reverse the in-plane electronic momentum. The influence of $T$ and $\sigma_{h}$ on the Hamiltonians of odd- $N$ multilayers is summarized as

$$
\begin{gathered}
\sigma_{h}: \sigma_{h} H[\mathbf{B}, U(x, y, z)] \sigma_{h}^{-1}=H[\mathbf{B}, U(x, y,-z)], \\
T: H^{*}[\mathbf{B}, U(\mathbf{r})]=H[-\mathbf{B}, U(\mathbf{r})] .
\end{gathered}
$$

Time reversal symmetry, Eq. (4), again ensures valley degeneracy of the electronic spectrum in the absence of an external magnetic field. The mirror reflection symmetry would seem to play the role of parity. However, it does not transform between states at the two valleys and is, therefore, unable to guarantee valley degeneracy in a magnetic field. It merely ensures that the spectrum at each valley is identical when the potential $U(\mathbf{r})$ is inverted with respect to $z=0$. Actually, we can show that Landau levels in odd-layered multilayer graphenes are approximately valley degenerate when $U(\mathbf{r})$ $=0$ by employing the effective-mass Hamiltonian described in the following section.

Although we focus on $A B A$ graphene multilayers in this paper, we point out that $A B C$ (rhombohedral) graphene multilayers, which have a different layer stacking, ${ }^{18,19}$ have inversion symmetry irrespective of their layer number and thus the valley degeneracy of Landau levels is always guaranteed.

\section{EFFECTIVE MASS HAMILTONIAN}

To investigate the Landau-level structure in detail, we adopt the effective-mass description of graphite in the Slonczewski-Weiss-McClure parameterization. ${ }^{20}$ We consider $A B$-stacked $N$-layer multilayer graphene in an external uniform magnetic field $\mathbf{B}$ perpendicular to the layer and with an external electrostatic potential $U_{j}$ at the $j$ th layer, which is uniform in the in-plane direction. In a basis with atomic components $\psi_{A 1}, \psi_{B 1}, \psi_{A 2}, \psi_{B 2}, \psi_{A 3}, \psi_{B 3}, \ldots$, the multilayer Hamiltonian at the $K_{\xi}$ valley ${ }^{13-15,21}$ is

$$
\mathcal{H}^{(\xi)}=\left(\begin{array}{cccccc}
H_{1} & V & & & & \\
V^{\dagger} & H_{2} & V^{\dagger} & & & \\
& V & H_{3} & V & & \\
& & V^{\dagger} & H_{4} & V^{\dagger} & \\
& & & \ddots & \ddots & \ddots
\end{array}\right),
$$

with

$$
\begin{gathered}
H_{i}=\left\{\begin{array}{cc}
\left(\begin{array}{cc}
U_{i} & v \pi^{\dagger} \\
v \pi & U_{i}+\delta
\end{array}\right) \quad(i \text { odd }) \\
\left(\begin{array}{cc}
U_{i}+\delta & v \pi^{\dagger} \\
v \pi & U_{i}
\end{array}\right) \quad(i \text { :even }),
\end{array}\right. \\
V=\left(\begin{array}{cc}
-v_{4} \pi^{\dagger} & v_{3} \pi \\
\gamma_{1} & -v_{4} \pi^{\dagger}
\end{array}\right) .
\end{gathered}
$$

Here $\pi=\xi \pi_{x}+i \pi_{y}, \quad \xi= \pm 1$ is the valley index, and $\boldsymbol{\pi}$ $=-i \hbar \nabla+e \mathbf{A}$ with the vector potential $\mathbf{A}$, which gives the external magnetic field as $\mathbf{B}=\nabla \times \mathbf{A}$. Parameter $U_{i}$ is the potential at the $i$ th layer, which is constant within each layer, and $\delta$ represents the energy difference between sites which have neighboring atoms right above or below them and those sites which do not and thus it only exists for $N \geq 2$. Parameter $v$ is the band velocity of monolayer graphene, which is written as $v=\sqrt{3} a \gamma_{0} / 2 \hbar$. Other velocities are defined as $v_{3}=\sqrt{3} a \gamma_{3} / 2 \hbar$ and $v_{4}=\sqrt{3} a \gamma_{4} / 2 \hbar$. We neglect parameters $\gamma_{2}$ and $\gamma_{5}$, which describe hopping between next-nearest neighboring layers. They actually break exact valley degeneracy as mentioned later.

For even- $N$ multilayers with $U_{j}=0$, the Hamiltonian Eq. (5) satisfies 


$$
\sigma_{2 N}^{\dagger} \mathcal{H}^{(\xi)} \sigma_{2 N}=\mathcal{H}^{(-\xi)},
$$

with $\sigma_{2 N}$ being a $2 N \times 2 N$ matrix

$$
\sigma_{2 N}=\left(\begin{array}{llll} 
& & & 1 \\
& & 1 & \\
& . & & \\
1 & & &
\end{array}\right),
$$

which is nothing but the inversion symmetry discussed in the previous section. In odd- $N$ multilayers with $U_{j}=0$, the Landau levels are degenerate in valleys due to a different symmetry. We can show this by employing a unitary transformation which decomposes the Hamiltonian of $N$-layer graphene, Eq. (5), into subsystems equivalent to monolayer and bilayer graphenes. We construct the basis as ${ }^{15,22}$

$$
\begin{aligned}
\left|\phi_{m}^{(X, \text { odd })}\right\rangle & =\sum_{j=1}^{N} f_{m}(j)\left|\psi_{X_{j}}\right\rangle, \\
\left|\phi_{m}^{(X, \text { even })}\right\rangle & =\sum_{j=1}^{N} g_{m}(j)\left|\psi_{X_{j}}\right\rangle,
\end{aligned}
$$

where $X=A, B$, and

$$
\begin{gathered}
f_{m}(j)=\frac{2}{\sqrt{N+1}} \sin \left(\frac{\pi}{2} j\right) \cos \left[\frac{m \pi}{2(N+1)} j\right], \\
g_{m}(j)=-\frac{2}{\sqrt{N+1}} \cos \left(\frac{\pi}{2} j\right) \sin \left[\frac{m \pi}{2(N+1)} j\right],
\end{gathered}
$$

where $j=1,2, \ldots, N$ is the layer index. The label $m$ is the subsystem index which ranges as

$$
m= \begin{cases}1,3,5, \ldots, N-1, \quad N=\text { even } \\ 0,2,4, \ldots, N-1, \quad N=\text { odd } .\end{cases}
$$

The superscript such as $(A$, odd $)$ indicates that the wavefunction has an amplitude only on $\left|A_{j}\right\rangle$ with odd $j$ 's.

When the Hamiltonian (5) with $U_{j}=0$ is written in the basis Eq. (9), the matrix is block diagonalized in each $m$. The case of $m=0$ is special in that $g_{m}(j)$ is identically zero, so that only two bases $\left\{\left|\phi_{0}^{(A, \text { odd })}\right\rangle,\left|\phi_{0}^{(B, \text { odd })}\right\rangle\right\}$ survive in Eq. (9). The submatrix is written for this two-component basis as

$$
\mathcal{H}_{m=0}^{(\xi)}=\left(\begin{array}{cc}
0 & v \pi^{\dagger} \\
v \pi & \delta
\end{array}\right),
$$

which is equivalent to the Hamiltonian of monolayer graphene except for the diagonal terms containing $\delta$. For $m \neq 0$, the submatrix for $\left\{\left|\phi_{m}^{(A, \text { odd })}\right\rangle,\left|\phi_{m}^{(B, \text { odd })}\right\rangle\right.$, $\left.\left|\phi_{m}^{(A, \text { even })}\right\rangle,\left|\phi_{m}^{(B, \text { even })}\right\rangle\right\}$ becomes

$$
\mathcal{H}_{m \neq 0}^{(\xi)}=\left(\begin{array}{cccc}
0 & v \pi^{\dagger} & -\lambda v_{4} \pi^{\dagger} & \lambda v_{3} \pi \\
v \pi & \delta & \lambda \gamma_{1} & -\lambda v_{4} \pi^{\dagger} \\
-\lambda v_{4} \pi & \lambda \gamma_{1} & \delta & v \pi^{\dagger} \\
\lambda v_{3} \pi^{\dagger} & -\lambda v_{4} \pi & v \pi & 0
\end{array}\right),
$$

where $\lambda \equiv \lambda_{m}$ is defined by

$$
\lambda_{m}=2 \cos \kappa_{m}, \quad \kappa_{m}=\frac{\pi}{2}-\frac{m \pi}{2(N+1)} .
$$

Equation (14) is identical to the Hamiltonian of bilayer graphene, except that interlayer-coupling parameters $\gamma_{1}, \gamma_{3}$, and $\gamma_{4}$ are multiplied by the factor $\lambda$.

Since the decomposed Hamiltonian matrices are analogous to those of monolayer or bilayer graphene, they obey the corresponding inversion symmetry. The bilayer-type submatrix, Eq. (14), obeys

$$
\sigma_{4}^{\dagger} \mathcal{H}_{m}^{(\xi)} \sigma_{4}=\mathcal{H}_{m}^{(-\xi)}
$$

which guarantees valley degeneracy of Landau levels. In the original basis, this "effective" inversion process Eq. (16) exchanges the wave amplitudes on odd-jth layers and those on even- $j$ th ones. In even- $N$ multilayers, the operation Eq. (16) for each eigenstate becomes equivalent to the original inversion symmetry Eq. (7) except for a phase factor. For the monolayer-type submatrix, Eq. (13) (exists in odd- $N$ ) satisfies

$$
\sigma_{2}^{\dagger} \mathcal{H}_{m=0}^{(\xi)} \sigma_{2} \approx \mathcal{H}_{m=0}^{(-\xi)},
$$

but it is only approximate since $\delta$ breaks this symmetry, unlike in the bilayer-type symmetry, Eq. (16). The operation of Eq. (17) exchanges the wave amplitudes within odd- $j$ layers.

The extra parameters $\gamma_{2}$ and $\gamma_{5}$ neglected here generally mix the states between different $m$ 's and also appear in diagonal elements within each $m .^{22}$ In odd- $N$ multilayers, they lift the valley degeneracy by breaking the effective inversion symmetry Eq. (16) through additional matrix elements. It should be noted that, in even- $N$ multilayers, valley degeneracy is never influenced by any extra lattice parameter because it is protected by inversion symmetry inherent in the lattice.

\section{LANDAU-LEVEL SPECTRA OF BILAYER AND TRILAYER GRAPHENE}

Here, we illustrate the valley degeneracy of the electronic spectra in even- and odd- $N$ multilayers through an analytical description of the Landau-level spectra of bilayer and trilayer graphenes. We use the magnetic length $\lambda_{B}=\sqrt{\hbar /(e B)}$ and an energy scale $\Gamma_{B}=\sqrt{2 \hbar v^{2} e B}=\sqrt{2} \hbar v / \lambda_{B}$ related to the inverse of the magnetic length. The Landau-level spectrum, in a magnetic field of magnitude $B$ perpendicular to the graphene sheet, may be found using the relation $\left(\pi, \pi^{\dagger}\right)$ $=\left(\sqrt{2} \hbar / \lambda_{B}\right)\left(a^{\dagger}, a\right)$ for $K_{+}$and $\left(\pi, \pi^{\dagger}\right)=\left(\sqrt{2} \hbar / \lambda_{B}\right)\left(a, a^{\dagger}\right)$ for $K_{-}{ }^{23}$ where $a^{\dagger}$ and $a$ are raising and lowering operators, respectively, which operate on the Landau-level wave function $\varphi_{n}$ as $a \varphi_{n}=\sqrt{n} \varphi_{n-1}$ and $a^{\dagger} \varphi_{n}=\sqrt{n+1} \varphi_{n+1}$.

Bilayer graphene. The Hamiltonian of bilayer graphene ${ }^{10,13,24,25}$ is given by the multilayer Hamiltonian, Eq. (5), with $N=2$. Here, we consider a simple form of the Hamiltonian that only contains nonzero parameters $\gamma_{0}$, describing nearest-neighbor intralayer hopping, $\gamma_{1}$, describing the dominant interlayer coupling, and the layer potential defined as $\left(U_{1}, U_{2}\right)=\Delta(1,-1)$. In the absence of interlayer asymmetry, $\Delta=0$, the spectrum consists of fourfold (valley 


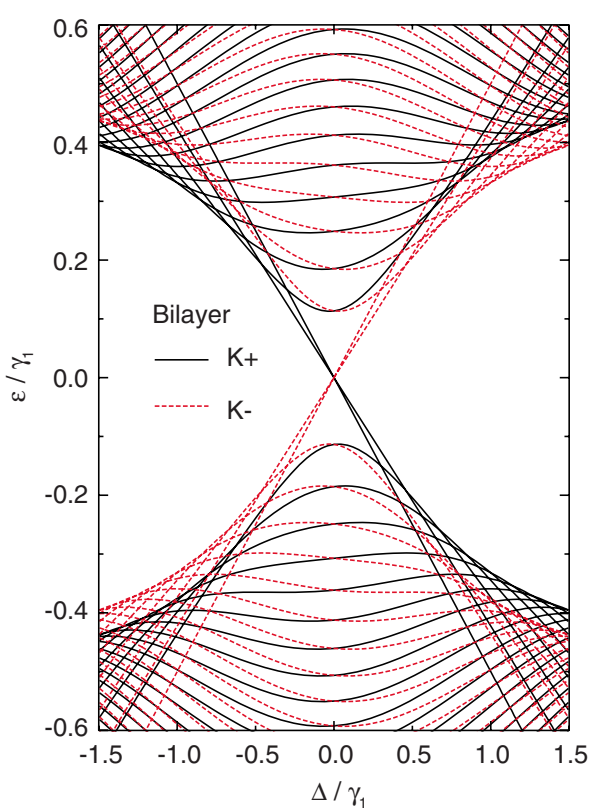

FIG. 2. (Color online) Low-energy Landau-level spectrum of bilayer graphene with $\Gamma_{B} / \gamma_{1}=0.3$ at each valley, plotted as a function of interlayer asymmetry $\Delta$.

and spin) degenerate Landau levels ${ }^{10}$ except for the level at zero energy which is eightfold degenerate. The valley degeneracy is guaranteed by spatial inversion symmetry Eq. (1). Finite $\Delta$ breaks spatial inversion symmetry and it splits the valley degeneracy of the levels, ${ }^{10,26-30}$ as indicated in the numerically calculated spectrum with a magnetic field of $\Gamma_{B} / \gamma_{1}=0.3$, plotted in Fig. $2 .{ }^{31}$ There, we can see that the energy levels of two valleys are related as $\varepsilon_{K_{+}}(\Delta)=\varepsilon_{K_{-}}(-\Delta)$ due to the inversion symmetry arguments presented in Sec. II. The valley splitting $\varepsilon_{K_{+}}(\Delta)-\varepsilon_{K_{-}}(\Delta)$ is thus an odd function in $\Delta$ and generally begins with a term linear in $\Delta$.

In the limit $\left\{\varepsilon, \sqrt{n} \Gamma_{B}, \Delta\right\} \ll \gamma_{1}$, the Hamiltonian is approximately described by an effective Hamiltonian ${ }^{10}$ operating in the space of two-component wave functions $\psi_{A 1}, \psi_{B 2}$,

$$
\begin{aligned}
\mathcal{H}_{A B}^{\text {(eff) }}= & -\frac{v^{2}}{\gamma_{1}}\left(\begin{array}{cc}
0 & \left(\pi^{\dagger}\right)^{2} \\
\pi^{2} & 0
\end{array}\right) \\
& +\Delta\left(\begin{array}{cc}
1-2 v^{2} \pi^{\dagger} \pi / \gamma_{1}^{2} & 0 \\
0 & -1+2 v^{2} \pi \pi^{\dagger} / \gamma_{1}^{2}
\end{array}\right) .
\end{aligned}
$$

The energy levels are obtained by assuming the wave function $\Psi_{n, K_{\xi}}=\left(c_{1} \varphi_{n+\xi}, c_{2} \varphi_{n-\xi}\right)$, where $\varphi_{m<0}$ is regarded as 0 . They are given by

$$
\varepsilon_{n \geq 1}= \pm \sqrt{\frac{\Gamma_{B}^{4}}{\gamma_{1}^{2}} n(n+1)+\Delta^{2}\left[1-\frac{2 \Gamma_{B}^{2}}{\gamma_{1}^{2}}\left(n+\frac{1}{2}\right)\right]^{2}}-\xi \frac{\Delta \Gamma_{B}^{2}}{\gamma_{1}^{2}},
$$

$$
\varepsilon_{0}=\xi \Delta\left(1-\frac{2 \Gamma_{B}^{2}}{\gamma_{1}^{2}}\right)
$$

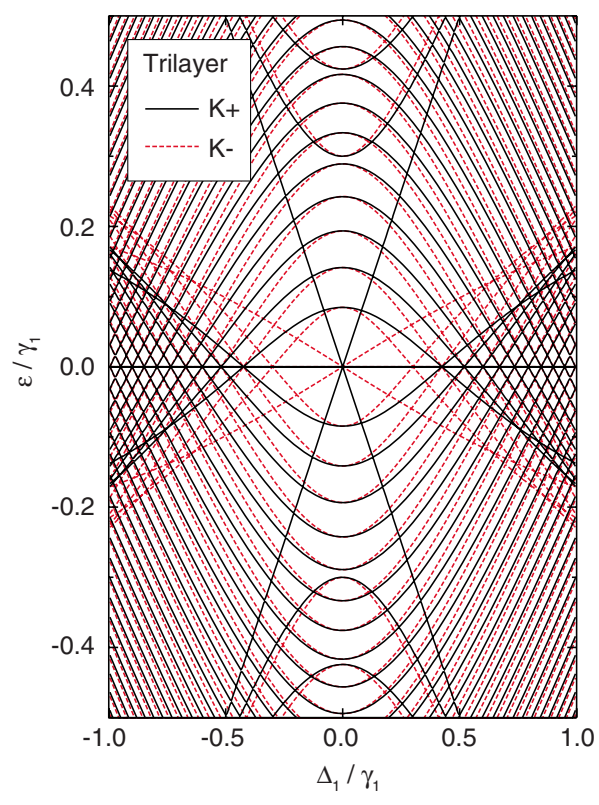

FIG. 3. (Color online) Low-energy Landau-level spectrum of $A B A$-stacked trilayer graphene with $\Gamma_{B} / \gamma_{1}=0.3$ at each valley, plotted as a function of interlayer asymmetry $\Delta_{1}$.

$$
\varepsilon_{-1}=\xi \Delta
$$

The splitting of the valley degeneracy in $n \geq 1$ (by terms containing $\xi$ ) is linear in the asymmetry potential $\Delta$ and also in the magnetic field $B$.

Trilayer graphene. The trilayer graphene $e^{12-15,21,32-36}$ Hamiltonian is given by the multilayer Hamiltonian, Eq. (5), with $N=3$. We again retain the parameters $\gamma_{0}$ and $\gamma_{1}$, and set the layer potential as $\left(U_{1}, U_{2}, U_{3}\right)=\Delta_{1}(1,0,-1)$, to focus our attention on interlayer asymmetry $\Delta_{1}=\left(U_{1}-U_{3}\right) / 2$. As described in Sec. III, we perform a unitary transformation to decompose the Hamiltonian into a monolayerlike part, Eq. (13), and a bilayerlike part, Eq. (14) (such a decomposed trilayer Hamiltonian is written explicitly in Ref. 32).

In the absence of interlayer asymmetry, $\Delta_{1}=0$, the spectrum consists of superimposed monolayerlike and bilayerlike spectra, ${ }^{13}$ with fourfold (valley and spin) degenerate Landau levels, except for the level at zero energy which is 12-fold degenerate. The valley degeneracy is guaranteed by the effective spatial inversion symmetry of the separate, monolayerlike and bilayerlike, parts of the decomposed Hamiltonian. Finite $\Delta_{1}$ breaks the effective spatial inversion symmetry and it splits the valley degeneracy of the levels as indicated in the numerically calculated spectrum at $\Gamma_{B} / \gamma_{1}=0.3$, plotted in Fig. $3 .{ }^{31}$ Unlike in bilayer, the energy spectrum at each valley is an even function of $\Delta_{1}$ due to the reflection symmetry argued in Sec. II. The valley splitting is therefore quadratic in $\Delta_{1}$, except for zero energy where the different levels are degenerate at each single valley and the energy level (and thus splitting) is allowed to be linear in $\Delta_{1}$.

In the limit $\left\{\varepsilon, \sqrt{n} \Gamma_{B}, \Delta_{1}\right\} \ll \gamma_{1}$, it is possible to obtain a simplified description of four electronic bands near zero energy, by eliminating atomic components associated with bands split away from zero by energy $\pm \sqrt{2} \gamma_{1}$, to obtain a 
four-component effective Hamiltonian in basis $\left[\psi_{A 1}\right.$ $\left.-\psi_{A 3}\right] / \sqrt{2},\left[\psi_{B 1}-\psi_{B 3}\right] / \sqrt{2},\left[\psi_{A 1}+\psi_{A 3}\right] / \sqrt{2}, \psi_{B 2}$,

$$
\mathcal{H}_{A B A}^{(\mathrm{eff})}=\left(\begin{array}{cccc}
0 & v \pi^{\dagger} & \Delta_{1} & 0 \\
v \pi & 0 & 0 & -\frac{\Delta_{1} v \pi^{\dagger}}{\sqrt{2} \gamma_{1}} \\
\Delta_{1} & 0 & 0 & -\frac{v^{2}\left(\pi^{\dagger}\right)^{2}}{\sqrt{2} \gamma_{1}} \\
0 & -\frac{\Delta_{1} v \pi}{\sqrt{2} \gamma_{1}} & -\frac{v^{2} \pi^{2}}{\sqrt{2} \gamma_{1}} & 0
\end{array}\right) .
$$

The energy levels are obtained by assuming the wave function $\Psi_{n, K_{\xi}}=\left(c_{1} \varphi_{n+\xi}, c_{2} \varphi_{n}, c_{3} \varphi_{n+\xi}, c_{4} \varphi_{n-\xi}\right)$. For levels with index $n \geq 1$ at valley $K_{+}$, the Hamiltonian Eq. (22) yields four energy levels for each $n$, given by

$$
\begin{gathered}
\varepsilon_{n \geq 1, K+}^{(u)}= \pm \sqrt{\Delta_{1}^{2}+(n+1) \Gamma_{B}^{2}}, \\
\varepsilon_{n \geq 1, K+}^{(l)}= \pm \frac{\sqrt{n} \Gamma_{B}}{\sqrt{2} \gamma_{1}} \frac{\left[\Delta_{1}^{2}-(n+1) \Gamma_{B}^{2}\right]}{\sqrt{\Delta_{1}^{2}+(n+1) \Gamma_{B}^{2}}} .
\end{gathered}
$$

For index $n=0$, there are three energy levels,

$$
\begin{gathered}
\varepsilon_{0, K+}^{(u)}= \pm \sqrt{\Delta_{1}^{2}+\Gamma_{B}^{2}}, \\
\varepsilon_{0, K+}^{(l)}=0,
\end{gathered}
$$

and, for $n=-1$, two energy levels,

$$
\varepsilon_{-1, K+}^{(u)}= \pm \Delta_{1} \text {. }
$$

Here, the superscript $(u)$ means that the level is related to an "upper" energy band which is split away from zero energy by $\Delta_{1}$ (i.e., the Landau-level energy approaches $\pm \Delta_{1}$ as $\Gamma_{B}$ $\rightarrow 0$ ), whereas superscript $(l)$ means that the level is related to a "lower" energy band near zero energy (i.e., the Landaulevel energy approaches zero as $\Gamma_{B} \rightarrow 0$ ).

The Landau-level spectrum at the second valley, $K_{-}$, differs as compared to that at $K_{+}$. For levels with index $n \geq 1$, the Hamiltonian Eq. (22) again yields four energy levels for each $n$ given by

$$
\begin{gathered}
\varepsilon_{n \geq 1, K-}^{(u)}= \pm \sqrt{\Delta_{1}^{2}+n \Gamma_{B}^{2}}, \\
\varepsilon_{n \geq 1, K-}^{(l)}= \pm \frac{\sqrt{n+1} \Gamma_{B}}{\sqrt{2} \gamma_{1}} \frac{\left[\Delta_{1}^{2}-n \Gamma_{B}^{2}\right]}{\sqrt{\Delta_{1}^{2}+n \Gamma_{B}^{2}}} .
\end{gathered}
$$

For index $n=0$, there are two energy levels at $K_{-}$,

$$
\varepsilon_{0, K-}^{(l)}= \pm \frac{\Delta_{1} \Gamma_{B}}{\sqrt{2} \gamma_{1}},
$$

and, for $n=-1$, one energy level,

$$
\varepsilon_{-1, K-}^{(l)}=0
$$

In the limit $\Delta_{1}=0$, the levels are degenerate in pairs of $\varepsilon_{n, K+}^{(u)}=\varepsilon_{n+1, K-}^{(u)}(n \geq 0)$ and $\varepsilon_{n, K+}^{(l)}=\varepsilon_{n, K-}^{(l)}(n \geq 1)$, which form monolayerlike and bilayerlike spectra, respectively. In the high-field limit, $\left|\Delta_{1}\right| \ll \Gamma_{B}$, the valley splitting of those levels is proportional to $\Delta_{1}^{2} / \gamma_{1}$ but independent of $B$, unlike bilayer graphene.

The other levels give degenerate zero-energy states at $\Delta_{1}=0$. For $K_{+}\left(K_{-}\right)$, one of the levels $\varepsilon_{-1, K+}^{(u)}\left(\varepsilon_{0, K-}^{(l)}\right)$ corresponds to the zero-energy level of the monolayerlike spectrum, whereas $\varepsilon_{0, K+}^{(l)}\left(\varepsilon_{-1, K-}^{(l)}\right)$ and one of $\varepsilon_{-1, K+}^{(u)}\left(\varepsilon_{0, K-}^{(l)}\right)$ correspond to two zero-energy levels of the bilayerlike spectrum. This yields an overall 12-fold degeneracy with spin degeneracy included (as opposed to the eightfold degeneracy in bilayers and fourfold degeneracy in monolayers). In the presence of finite interlayer asymmetry, two of the otherwisezero levels at each valley are hybridized and split away from zero [Eq. (27) at $K_{+}$and Eq. (30) at $K_{-}$], whereas one level at each valley remains at zero [Eq. (26) at $K_{+}$and Eq. (31) at $K_{-}$]. Zero-energy states are formed primarily by different atomic orbitals at different valleys and, as in bilayers, ${ }^{10,13,37}$ the orbitals in trilayers are on different layers, so that those levels exhibit quite different dependences on the interlayer asymmetry between $K_{+}$and $K_{-}$.

In realistic experimental situations, interlayer potential asymmetry can be produced by gate-induced electric fields. There, the potentials $U_{i}$, which are taken as external parameters in the present work, should be determined selfconsistently including the screening effect of graphene electrons. ${ }^{24,32}$ The self-consistent Landau-level structure and quantum Hall effect as a function of gate voltage would be an important future study. ${ }^{38}$

It has been pointed out ${ }^{38}$ that a number of level crossings will occur in bilayer graphene at finite asymmetry (Fig. 2). We note that the Landau-level spectrum of trilayer graphene (Fig. 3) has a very rich pattern of level crossings at a range of different values of energy and asymmetry. The presence of additional terms in the Hamiltonian will tend to produce some anticrossings in the spectrum. The precise position and nature of level crossings in the spectra of multilayer graphenes will be the subject of future investigation.

\section{ACKNOWLEDGMENTS}

The authors thank T. Ando and M. Mucha-Kruczyński for discussions. This project has been funded by EPSRC First Grant No. EP/E063519/1, the Royal Society, the Daiwa Anglo-Japanese Foundation, and by Grants-in-Aid for Scientific Research from the Ministry of Education, Culture, Sports, Science and Technology, Japan. 
${ }^{1}$ K. S. Novoselov, A. K. Geim, S. V. Morozov, D. Jiang, Y. Zhang, S. V. Dubonos, I. V. Grigorieva, and A. A. Firsov, Science 306, 666 (2004).

${ }^{2}$ K. S. Novoselov, A. K. Geim, S. V. Morozov, D. Jiang, M. I. Katsnelson, I. V. Grigorieva, S. V. Dubonos, and A. A. Firsov, Nature (London) 438, 197 (2005).

${ }^{3}$ Y. B. Zhang, Y. W. Tan, H. L. Stormer, and P. Kim, Nature (London) 438, 201 (2005).

${ }^{4}$ K. S. Novoselov, E. McCann, S. V. Morozov, V. I. Fal'ko, M. I. Katsnelson, U. Zeitler, D. Jiang, F. Schedin, and A. K. Geim, Nat. Phys. 2, 177 (2006).

${ }^{5}$ G. W. Semenoff, Phys. Rev. Lett. 53, 2449 (1984).

${ }^{6}$ T. Ando, T. Nakanishi, and R. Saito, J. Phys. Soc. Jpn. 67, 2857 (1998).

${ }^{7}$ V. P. Gusynin, S. G. Sharapov, and J. Carbotte, Int. J. Mod. Phys. B 21, 4611 (2007).

${ }^{8}$ Y. Zhang, Z. Jiang, J. P. Small, M. S. Purewal, Y.-W. Tan, M. Fazlollahi, J. D. Chudow, J. A. Jaszczak, H. L. Stormer, and P. Kim, Phys. Rev. Lett. 96, 136806 (2006).

${ }^{9}$ J. L. Manes, F. Guinea, and M. A. H. Vozmediano, Phys. Rev. B 75, 155424 (2007).

${ }^{10}$ E. McCann and V. I. Fal'ko, Phys. Rev. Lett. 96, 086805 (2006).

${ }^{11}$ T. Ohta, A. Bostwick, T. Seyller, K. Horn, and E. Rotenberg, Science 313, 951 (2006).

${ }^{12}$ S. Latil and L. Henrard, Phys. Rev. Lett. 97, 036803 (2006).

${ }^{13}$ F. Guinea, A. H. Castro Neto, and N. M. R. Peres, Phys. Rev. B 73, 245426 (2006).

${ }^{14}$ B. Partoens and F. M. Peeters, Phys. Rev. B 74, 075404 (2006); 75, 193402 (2007).

${ }^{15}$ M. Koshino and T. Ando, Phys. Rev. B 76, 085425 (2007); 77, 115313 (2008).

${ }^{16}$ H. Min and A. H. MacDonald, Phys. Rev. B 77, 155416 (2008).

${ }^{17}$ Corners of the hexagonal Brillouin zone are located at wave vector $\mathbf{K}_{\xi}=\xi\left(\frac{4}{3} \pi a^{-1}, 0\right)$, where $\xi= \pm 1$ and $a$ is the lattice constant.

${ }^{18}$ R. R. Haering, Can. J. Phys. 36, 352 (1958).

${ }^{19}$ J. W. McClure, Carbon 7, 425 (1969).

${ }^{20}$ M. S. Dresselhaus and G. Dresselhaus, Adv. Phys. 51, 1 (2002).
${ }^{21}$ C. L. Lu, C. P. Chang, Y. C. Huang, R. B. Chen, and M. L. Lin, Phys. Rev. B 73, 144427 (2006).

${ }^{22}$ M. Koshino and T. Ando, Solid State Commun. 149, 1123 (2009).

${ }^{23}$ The Quantum Hall Effect, edited by R. E. Prange and S. M. Girvin (Springer-Verlag, New York, 1986).

${ }^{24}$ E. McCann, Phys. Rev. B 74, 161403(R) (2006).

${ }^{25}$ M. Koshino and T. Ando, Phys. Rev. B 73, 245403 (2006).

${ }^{26}$ E. V. Castro, K. S. Novoselov, S. V. Morozov, N. M. R. Peres, J. M. B. Lopes dos Santos, J. Nilsson, F. Guinea, A. K. Geim, and A. H. Castro Neto, Phys. Rev. Lett. 99, 216802 (2007).

${ }^{27}$ M. Koshino, Phys. Rev. B 78, 155411 (2008).

${ }^{28}$ M. Mucha-Kruczyński, D. S. L. Abergel, E. McCann, and V. I. Fal'ko, J. Phys.: Condens. Matter 21, 344206 (2009).

${ }^{29}$ M. Mucha-Kruczyński, E. McCann, and V. I. Fal'ko, Solid State Commun. 149, 1111 (2009).

${ }^{30}$ M. Nakamura, E. V. Castro, and B. Dóra, Phys. Rev. Lett. 103, 266804 (2009).

${ }^{31}$ Note that charge-conjugation symmetry (Ref. 7) of the lowenergy effective Hamiltonians of monolayer, bilayer, and multilayer graphene ensures electron-hole symmetry of the spectra that, depending on whether $N$ is odd or even, occurs between spectra at either the same or at opposite valleys, even in the presence of finite interlayer asymmetry.

${ }^{32}$ M. Koshino and E. McCann, Phys. Rev. B 79, 125443 (2009).

${ }^{33}$ T. Ohta, A. Bostwick, J. L. McChesney, T. Seyller, K. Horn, and E. Rotenberg, Phys. Rev. Lett. 98, 206802 (2007).

${ }^{34}$ J. Güttinger, C. Stampfer, F. Molitor, D. Graf, T. Ihn, and K. Ensslin, New J. Phys. 10, 125029 (2008).

${ }^{35}$ M. F. Craciun, S. Russo, M. Yamamoto, J. B. Oostinga, A. F. Morpurgo, and S. Tarucha, Nat. Nanotechnol. 4, 383 (2009).

${ }^{36}$ M. Aoki and H. Amawashi, Solid State Commun. 142, 123 (2007).

${ }^{37}$ M. Koshino and E. McCann, Phys. Rev. B 80, 165409 (2009).

${ }^{38}$ L. M. Zhang, M. M. Fogler, D. P. Arovas, and F. Guinea, APS March Meeting, 2010, http://meetings.aps.org/Meeting/ MAR10/Event/120799 